\title{
Thermal infrared observations of the Hayabusa spacecraft target asteroid 25143 Itokawa ${ }^{\star}$
}

\author{
T. G. Müller ${ }^{1}$, T. Sekiguchi ${ }^{2}$, M. Kaasalainen ${ }^{3}$, M. Abe ${ }^{4}$, and S. Hasegawa ${ }^{4}$
}

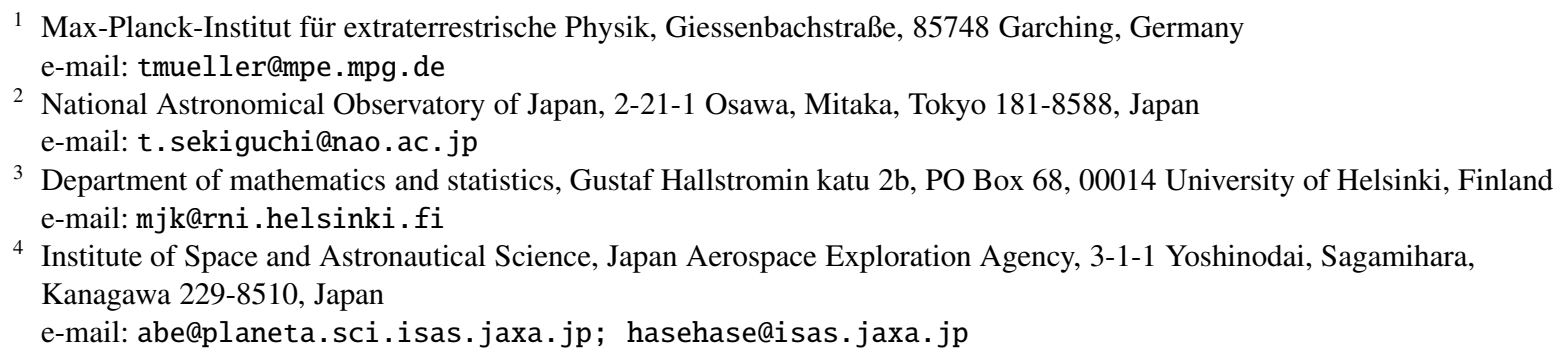

Received 20 July 2005 / Accepted 29 August 2005

\section{ABSTRACT}

We obtained $N$ - and $Q$-band observations of the Apollo-type asteroid 25143 Itokawa during its close Earth approach in July 2004 with TIMMI2 at the ESO $3.6 \mathrm{~m}$ telescope. Our photometric measurement, in combination with already published data, allowed us to derive a radiometric effective diameter of $0.32 \pm 0.03 \mathrm{~km}$ and an albedo of $0.19_{-0.03}^{+0.11}$ through a thermophysical model. This effective diameter corresponds to a slightly asymmetrical and flattened ellipsoid of the approximate size of $520( \pm 50) \times 270( \pm 30) \times 230( \pm 20) \mathrm{m}$, based on the Kaasalainen et al. (2005, Proceedings of the 1st Hayabusa Symposium, ASP Conf. Ser., submitted) shape model. Our studies show that the thermal observations lead to size estimates which are about $15 \%$ smaller than the radar results (Ostro et al. 2005, Met. Plan. Sci., submitted), slightly outside the stated radar uncertainties of $\pm 10 \%$. We determined a rather high thermal inertia of $750 \mathrm{~J} \mathrm{~m}^{-2} \mathrm{~s}^{-0.5} \mathrm{~K}^{-1}$. This is an indication for a bare rock dominated surface, a thick dust regolith can be excluded as well as a metallic surface. From our data we constructed a $10.0 \mu \mathrm{m}$ thermal lightcurve which is nicely matched in amplitude and phase by the shape and spin vector solution in combination with our TPM description. The assumed S-type bulk density in combination with radiometric size lead to a total mass estimate of $4.5_{-1.8}^{+2.0} \times 10^{10} \mathrm{~kg}$.

Key words. minor planets, asteroids - radiation mechanisms: thermal - infrared: solar system

\section{Introduction}

The Near-Earth asteroid (NEA) 25143 Itokawa (1998 SF36) is the target for the Japanese Hayabusa (MUSES-C) sample return mission. The spacecraft will arrive at the asteroid in summer 2005 and hover close to the surface for about 3 months before it will collect surface samples which will be brought back to Earth in June 2007.

Several ground-based observing campaigns took place during the last years to derive various properties of Itokawa. E.g., Binzel et al. (2001) concluded from visible and near-infrared spectroscopic measurements that the spectral characteristics (S(IV)-type) match the LL ordinary chondrite class meteorites. Ostro et al. (2004, 2005) report on delay-Doppler images obtained at Arecibo and Goldstone, resulting in size, shape, radar

^ Based on observations collected at the European Southern Observatory, Chile; ESO, No. 73.C-0772. albedo and surface roughness estimates. Kaasalainen et al. (2003, 2005) determined through lightcurve inversion techniques a high quality shape and pole solution together with a period of $P_{\text {sid }}=12.13237 \pm 0.00008 \mathrm{~h}$. Sekiguchi et al. (2003) observed Itokawa at thermal infrared wavelengths and used the powerful radiometric technique (e.g. Harris \& Lagerros 2002) to determine a size of $0.35 \pm 0.03 \mathrm{~km}$ and an albedo of $0.23(+0.07,-0.05)$. Müller et al. (2004) reported on thermal property studies of Itokawa based on multi-epoch thermal infrared photometric data. But their data set did not allow to find a unique solution for the size, thermal inertia and roughness. Based on the radar size, they derived a thermal inertia between 5 and 10 times that of the Moon.

Here, we used all available thermal infrared data together with own observations, taken during a close approach in July 2004 (Sect. 2). The modelling and the derivation of thermophysical properties of Itokawa was then performed through the well-established, tested and frequently used thermophysical model (TPM) by Lagerros (1996, 1997, 1998; see Sects. 3-5). 
Table 1. Summary of TIMMI2 observations of asteroid 25143 Itokawa. The phase angles are positive before opposition and negative after. We added data from Sekiguchi et al. (2003) and Delbo (2004).

\begin{tabular}{|c|c|c|c|c|c|c|c|}
\hline \multirow{2}{*}{$\frac{\text { No }}{01}$} & \multicolumn{2}{|c|}{$\begin{array}{l}\text { Mid-time } \\
\text { (Day UT) }\end{array}$} & \multirow{2}{*}{$\begin{array}{l}\begin{array}{l}\text { Filter } \\
\text { band }\end{array} \\
\text { N11.9 }\end{array}$} & \multirow{2}{*}{$\begin{array}{l}r \\
{[\mathrm{AU}]} \\
1.059232\end{array}$} & \multirow{2}{*}{$\begin{array}{l}\Delta \\
{[\mathrm{AU}]} \\
0.073897\end{array}$} & \multirow{2}{*}{$\begin{array}{l}\alpha \\
{\left[{ }^{\circ}\right]} \\
+27.54\end{array}$} & \multirow{2}{*}{$\begin{array}{l}\text { Remarks } \\
\text { Sekiguchi et al. (2003) }\end{array}$} \\
\hline & 2001/Mar./14 & $05: 50$ & & & & & \\
\hline 02 & 2001/Apr./8 & $09: 27$ & $N 11.9$ & 0.983221 & 0.053606 & 108.33 & Delbo (2004) \\
\hline 03 & 2001/Apr./8 & $09: 42$ & $N 10.4$ & 0.983198 & 0.053633 & 108.35 & and priv. comm. \\
\hline 04 & 2001/Apr./8 & $10: 01$ & $N 12.9$ & 0.983169 & 0.053667 & 108.37 & $"$ \\
\hline 05 & 2001/Apr./8 & $10: 18$ & $N 8.9$ & 0.983142 & 0.053698 & 108.38 & ” \\
\hline 06 & 2001/Apr./8 & $10: 34$ & $N 11.9$ & 0.983117 & 0.053728 & 108.40 & " \\
\hline 07 & 2001/Apr./9 & $09: 28$ & $N 12.9$ & 0.981024 & 0.056409 & 109.93 & " \\
\hline 08 & 2001/Apr./9 & $09: 45$ & $N 9.8$ & 0.980999 & 0.056441 & 109.95 & $"$ \\
\hline 09 & 2001/Apr./9 & $10: 03$ & $N 10.4$ & 0.980972 & 0.056475 & 109.96 & ” \\
\hline 10 & 2001/Apr./9 & $10: 18$ & $N 11.9$ & 0.980949 & 0.056504 & 109.98 & $"$ \\
\hline 11 & 2001/Apr./9 & $10: 32$ & $N 11.9$ & 0.980928 & 0.056530 & 109.99 & $"$ \\
\hline 12 & 2004/Jul./1 & 06:03 & $N 1$ & 1.028243 & 0.020164 & -54.63 & this work \\
\hline 13 & 2004/Jul./1 & $06: 19$ & $N 1$ & 1.028279 & 0.020193 & -54.56 & $"$ \\
\hline 14 & 2004/Jul./1 & $06: 36$ & $N 1$ & 1.028318 & 0.020224 & -54.49 & ” \\
\hline 15 & 2004/Jul./1 & $06: 54$ & $N 1$ & 1.028359 & 0.020257 & -54.41 & $"$ \\
\hline 16 & 2004/Jul./1 & $07: 16$ & $N 2$ & 1.028409 & 0.020298 & -54.31 & $"$ \\
\hline 17 & 2004/Jul./1 & $07: 36$ & $N 2$ & 1.028454 & 0.020335 & -54.22 & $"$ \\
\hline 18 & 2004/Jul./1 & $07: 53$ & $N 12.9$ & 1.028492 & 0.020367 & -54.15 & $"$ \\
\hline 19 & 2004/Jul./1 & 08:09 & $N 12.9$ & 1.028529 & 0.020397 & -54.08 & $"$ \\
\hline 20 & 2004/Jul./1 & $08: 37$ & $Q 1$ & 1.028592 & 0.020450 & -53.95 & $"$ \\
\hline
\end{tabular}

The results are discussed in the context of the already known properties of Itokawa (Sect. 6) and conclusions are drawn in Sect. 7.

\section{Observations and data reduction}

We combined the observations from Sekiguchi et al. (2003) (data set \#1 in Table 1), with the data set from Delbo (2004) (data set \#2), and our own observations (data set \#3). All measurements were taken with the TIMMI2 instrument (Käufl et al. 2003) at the ESO La Silla $3.6 \mathrm{~m}$ telescope. Table 1 summarises the observing geometries for all 20 measurements.

A standard chopping and nodding technique was utilized for all observations to reduce the atmospheric and telescope background emission. Chop and nod throws were $10^{\prime \prime}$, respectively. For the imaging observations, a pixel scale of $0.2^{\prime \prime}$ was chosen, and on source integration times were $22 \mathrm{~min}$ (obs \#1), 10-13 min (obs \#2 to \#11), $7.5 \mathrm{~min}$ (obs \#12 to \#19), and 25 min (obs \#20).

Both already published data sets, from Sekiguchi et al. (2003) and from Delbo (2004), were re-calibrated using the official central filter wavelengths in combination with the flux densities of the corresponding stellar models (see Table 2).

A more detailed data reduction and calibration was performed on our own data set. Unfortunately, the telescope tracking on 25143 Itokawa was not perfect in the 2004 observing run. This had the consequence that the pipeline-reduced images showed elongated sources (see left side of Fig. 1). Therefore, we took the raw images and processed them in a pipelinelike manner together with a fixed $x-y-$ shift rate of typically 0.5 to 1.0 pixels $/ \mathrm{min}$. A centroid determination in combination with a standard shift-and-add technique was not possible due to the faint sources which are not always visible on individual raw frames. The results of this process are illustrated on the right side of Fig. 1.

We applied standard aperture photometry on the tracking corrected images with same apertures on the stars and asteroids. The aperture radii were chosen with respect to the growth curves (for details see e.g. Delbo 2004). All four on-array signatures of the sources were used for the flux calibration. Colour differences between stars and 25143 Itokawa were negligible (about 1-3\%) for the used filters in combination with the atmospheric transmission at $\mathrm{La}$ Silla (http://www.ls.eso.org/lasilla/sciops/3p6/timmi/ html/AtmosphericTransm.html).

The observational results are summarised in Table 3 .

The error values of observations \#12 to \#20 include the uncertainties of the calibration star models (3\% in $N$-band and $4 \%$ in $Q$-band), the aperture photometry error (N1: $3 \%$, $N 2: 2 \%, N 12.9: 2 \%, Q 1: 10-20 \%$ ) and an error for the flat-field residuals (about $3-6 \%$ in $N$ and about $10-15 \%$ for $Q$-band, depending on the relative placement of the calibrator and the asteroid on the TIMMI2 array). The error calculations of observations \#1 to \#11 are described in the corresponding references. 
Table 2. Monochromatic flux densities in $[\mathrm{Jy}]$ of the stellar calibrators at the TIMMI 2 central filter wavelengths. Note: The key wavelengths were taken from http://www.ls.eso.org/lasilla/sciops/3p6/timmi/Filters. The model fluxes were taken from http://www.iso.vilspa.esa.es/users/expl_lib/ISO/wwwcal/isoprep/cohen/templates/ and interpolated to the central filter wavelengths.

\begin{tabular}{lllllllll}
\hline \hline \multicolumn{8}{c}{ Filter/Wavelength $[\mu \mathrm{m}]$} \\
\hline Star & $N 1$ & $N 8.9$ & $N 9.8$ & $N 10.4$ & $N 2$ & $N 11.9$ & $N 12.9$ & $Q 1$ \\
& 8.70 & 8.73 & 9.68 & 10.38 & 10.68 & 11.66 & 12.35 & 17.72 \\
\hline HD 47105 & 9.16 & 9.08 & 7.47 & 6.52 & 6.17 & 5.19 & 4.58 & 2.27 \\
HD 123139 & 69.97 & 69.51 & 57.37 & 50.09 & 47.61 & 40.08 & 35.59 & 17.63 \\
HD 196171 & 25.58 & 25.42 & 20.98 & 18.32 & 17.41 & 14.65 & 13.01 & 6.45 \\
\hline
\end{tabular}
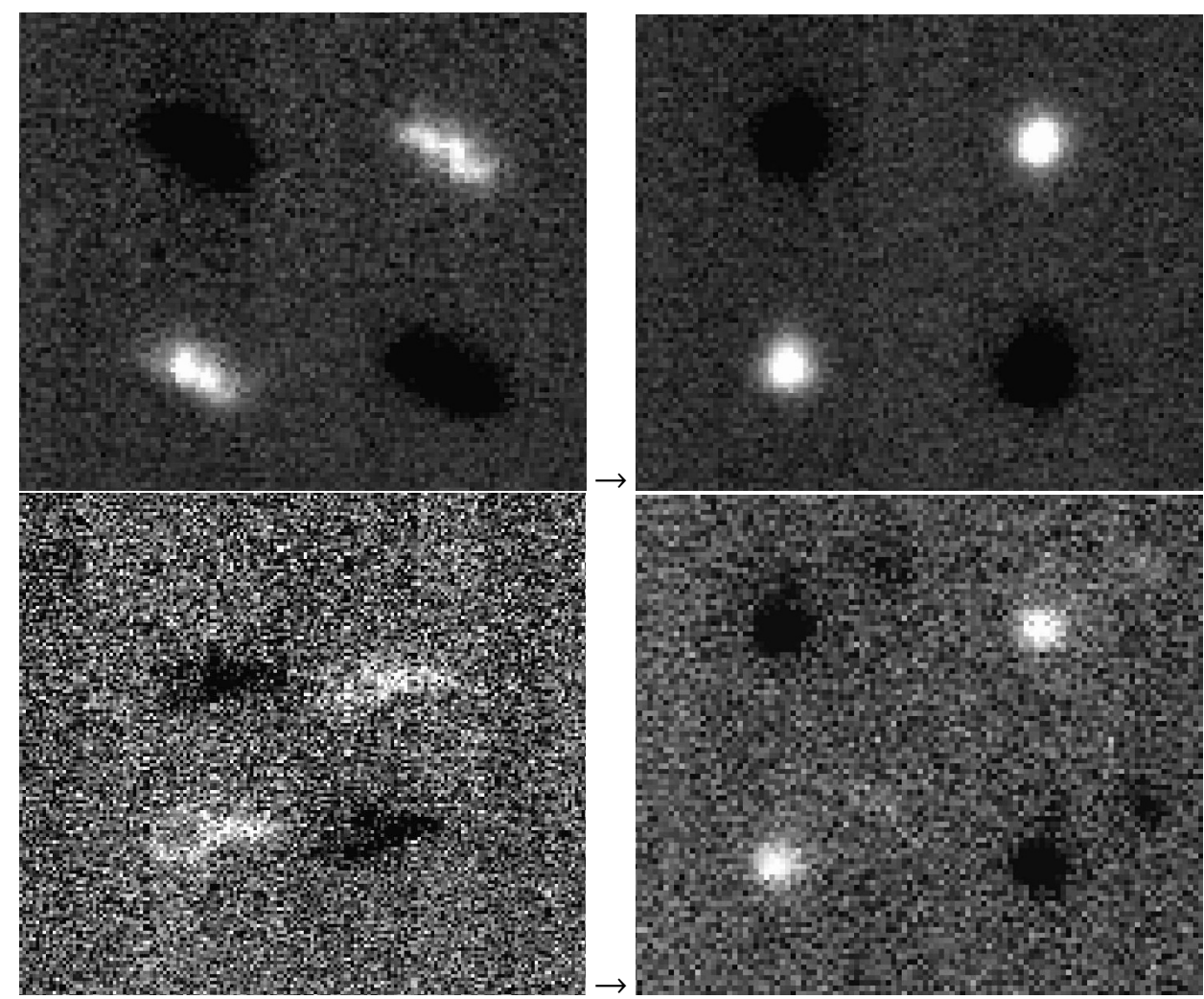

Fig. 1. Pipeline-processing in comparison with re-reduced asteroid images. Top: Itokawa measurement \#12 (Table 1), N1-filter, 7.5 min integration time, left: pipeline-product, right: the 60 raw images were shifted by 1.0 pixels per minute in $x$-direction and by -1.0 pixels per minute in $y$-direction and then co-added. Bottom: Itokawa measurement \#20 (Table 1), Q1-filter, 25.8 min integration time, left: pipeline-product, right: the 234 raw images were shifted by 0.8 pixels per minute in $x$-direction and by 0.2 pixels per minute in $y$-direction and then co-added.

\section{Thermophysical model description}

We applied the TPM by Lagerros $(1996,1997,1998)$ to all 20 measurements from Table 3 to investigate the physical and thermal properties of Itokawa. On the large scale, the TPM considers the asteroid size, the global shape and spin vector and the actual observing and illumination geometry at the time of an observation. On the small micrometer scale, the TPM takes into account the reflected, absorbed and emitted energy, and also the heat conduction into the surface regolith. The albedo and emissivity control the energy balance and thereby the surface temperature. The thermal inertia in combination with the rotation period and the orientation of the spin vector influence the diurnal temperature variations. As a result, the thermal inertia is strongly connected to the interpretation of mid-IR observations, namely when comparing before and after opposition observations at large phase angles with very different temperatures of the terminator. Moreover, the thermal inertia determines the amplitude of the thermal lightcurve for a given aspect angle. The TPM beaming model, described by $\rho$, the rms of the surface slopes and $f$, the fraction of the surface covered by craters, accounts for the non-isotropic heat radiation, noticeable at phase angles close to opposition. But it also influences the shape of the spectral energy distribution in the mid-IR. Detailed considerations of the $\Gamma, \rho$ and $f$ influences for various observing geometries and wavelengths are discussed in e.g., Müller (2002) or Dotto et al. (2000). 
Table 3. Summary of TIMMI 2 observational results for asteroid 25143 Itokawa. Note: The Sekiguchi et al. (2003) and Delbo (2004) fluxes and errors have been recalculated for the true central filter wavelengths, using the corresponding stellar fluxes. Measurements from April 9, 2001 (marked with $\star$ ) were taken under less favorable conditions in comparison with data from April 8 (M. Delbo, priv. comm.). Our new observations are listed under \#12 until \#20.

\begin{tabular}{|c|c|c|c|c|c|}
\hline No & Filter & $\begin{array}{r}\lambda_{\mathrm{c}} \\
{[\mu \mathrm{m}]}\end{array}$ & $\begin{array}{l}\text { FD } \\
{[\mathrm{Jy}]}\end{array}$ & $\begin{array}{l}\sigma_{\text {err }} \\
{[\mathrm{Jy}]}\end{array}$ & Remarks \\
\hline 01 & $N 11.9$ & 11.66 & 0.264 & \pm 0.044 & $\begin{array}{l}\text { Sekiguchi et al. (2003) } \\
\text { (re-calibrated) }\end{array}$ \\
\hline 02 & $N 11.9$ & 11.66 & 0.164 & \pm 0.021 & Delbo (2004) \\
\hline 03 & $N 10.4$ & 10.38 & 0.144 & \pm 0.018 & and priv. comm. \\
\hline 04 & $N 12.9$ & 12.35 & 0.170 & \pm 0.022 & (re-calibrated) \\
\hline 05 & $N 8.9$ & 8.73 & 0.086 & \pm 0.022 & $"$ \\
\hline 06 & $N 11.9$ & 11.66 & 0.149 & \pm 0.019 & $"$ \\
\hline 07 & $N 12.9$ & 12.35 & 0.258 & \pm 0.032 & $"(\star)$ \\
\hline 08 & $N 9.8$ & 9.68 & 0.108 & \pm 0.016 & $"(\star)$ \\
\hline 09 & $N 10.4$ & 10.38 & 0.169 & \pm 0.027 & $"(\star)$ \\
\hline 10 & $N 11.9$ & 11.66 & 0.242 & \pm 0.030 & $"(\star)$ \\
\hline 11 & $N 11.9$ & 11.66 & 0.193 & \pm 0.028 & $"(\star)$ \\
\hline 12 & $N 1$ & 8.73 & 1.92 & \pm 0.15 & this work \\
\hline 13 & $N 1$ & 8.73 & 1.97 & \pm 0.16 & $"$ \\
\hline 14 & $N 1$ & 8.73 & 1.75 & \pm 0.14 & $"$ \\
\hline 15 & $N 1$ & 8.73 & 1.67 & \pm 0.13 & $"$ \\
\hline 16 & $N 2$ & 10.68 & 1.94 & \pm 0.14 & " \\
\hline 17 & $N 2$ & 10.68 & 1.89 & \pm 0.13 & $"$ \\
\hline 18 & $N 12.9$ & 12.35 & 2.17 & \pm 0.13 & $"$ \\
\hline 19 & $N 12.9$ & 12.35 & 1.80 & \pm 0.11 & $"$ \\
\hline 20 & $Q 1$ & 17.72 & 2.49 & \pm 0.50 & $"$ \\
\hline
\end{tabular}

An $H_{\mathrm{V}}$-value of 19.9 (Kaasalainen et al. 2003; M. Abe, priv. comm.; Sekiguchi et al. 2003) and a $G$-value of 0.21 (Abe et al. $2002 \mathrm{a}, \mathrm{b}$ ) was used to describe the visual brightness of Itokawa. We assumed a constant emissivity of 0.9 at all wavelength.

The shape-model used here (Kaasalainen et al. 2005) is an update and refinement of the model presented in Kaasalainen et al. (2003). The model accommodates new photometric observations from December 2003 to September 2004, as well as some 2001 data additional to the 2000-2001 apparition dataset presented in Kaasalainen et al. (2003). The long time-line of the updated dataset allowed accurate period determination for Itokawa, and a refined pole and shape estimate. All parts of Itokawa's surface were well visible during the two apparitions; however, the long period precluded fully covered rotational phases for single lightcurves. Calibrated photometry allowed the determination of Itokawa's solar phase curve for a wide range of solar phase angles. The refined rotation parameters are $\beta=-89^{\circ} \pm 5^{\circ}, \lambda=330^{\circ}$ for the ecliptic latitude and longitude of the pole, and $P=12.13237 \pm 0.00008 \mathrm{~h}$ for the sidereal period. Figure 2 shows equatorial edge-on and pole-on images of the shape model. The model agrees well with the radar-based one (Ostro et al. 2005).

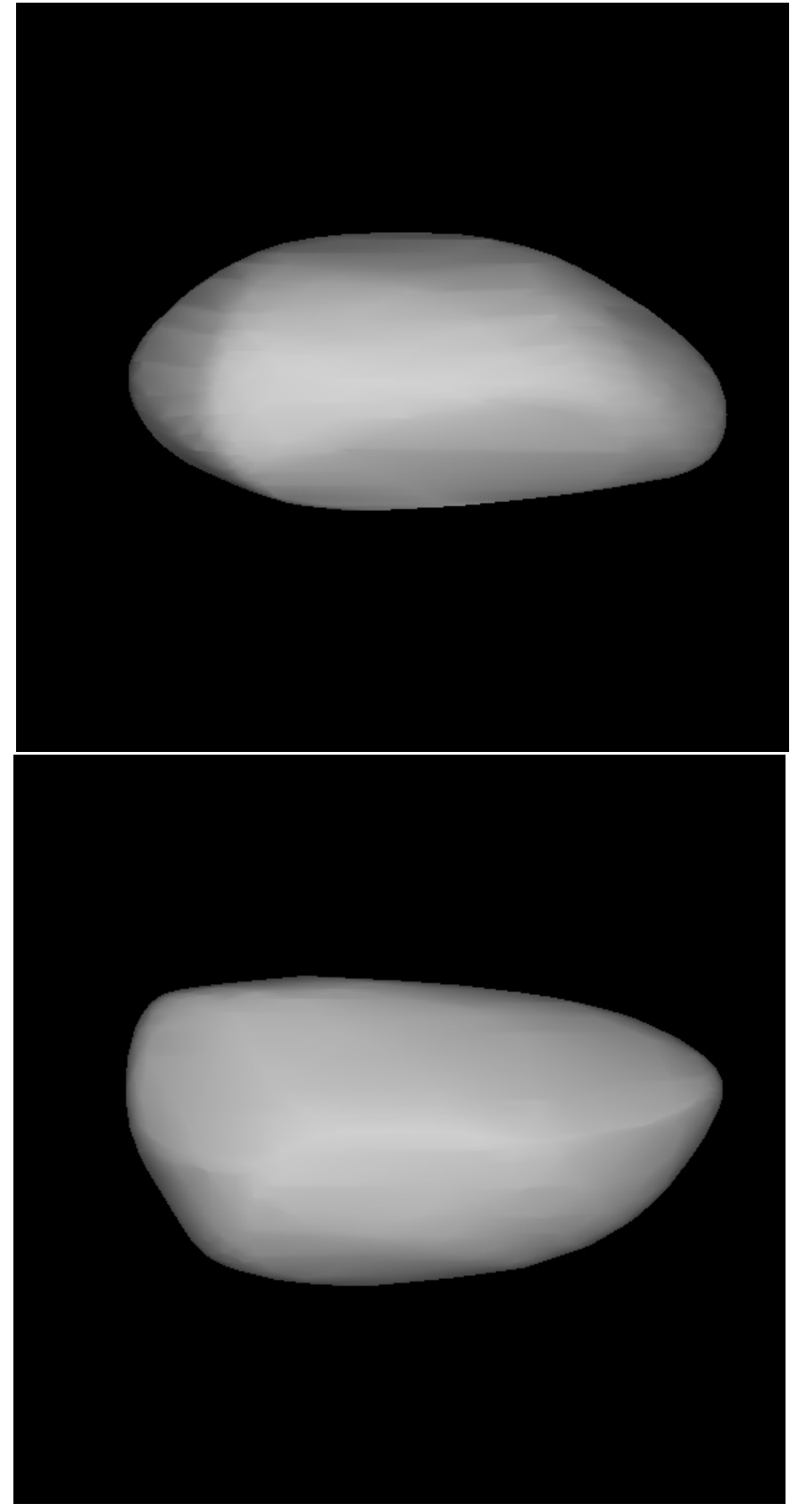

Fig. 2. Equatorial edge-on (top) and pole-on (bottom) images of the shape model.

\section{Thermophysical modelling on the combined dataset}

Our data set is very homogenous with respect to the used instrument settings, observing technique, data reduction and calibration scheme. The data cover a wide range of different observing geometries, including before and after opposition, at different wavelengths and rotational phases. Therefore, it was possible to adjust the thermal inertia and beaming parameters to see how these variations influence the calculation of the radiometric diameter and albedo solutions. The investigations of the resulting diameters and albedos give clues about the optimal model parameters. Only specific model 
parameters will allow that e.g. the data taken at very different phase angles before and after opposition will result in the same radiometric diameter and albedo solutions. Another quality criteria is that the resulting diameter and albedo values show no trends with wavelengths or rotational phase. The goal was to find the best diameter/albedo solution with the smallest standard deviations which fits all 20 measurements. A similar procedure was already used by Müller \& Lagerros (1998, 2002) for several main-belt asteroids. For large, regolith covered asteroids, the least-square process gave typical thermal inertias of $10-15 \mathrm{~J} \mathrm{~m}^{-2} \mathrm{~s}^{-0.5} \mathrm{~K}^{-1}$ and beaming parameters of $\rho=0.7$ and $f=0.6$ (Müller et al. 1999).

As a first step, we tried to find out how the thermal inertia influences the determination of the radiometric diameter/albedo solutions. For observations taken at very large phase angles the thermal inertia is the most important parameter for a consistent diameter and albedo determination. This is due to the non-zero temperature of the large terminator which contributes significantly to the disk-integrated flux. The thermal inertia $\Gamma$ was varied in a physically meaningful range between 0 and $2500 \mathrm{~J} \mathrm{~m}^{-2} \mathrm{~s}^{-0.5} \mathrm{~K}^{-1}$. Where $\Gamma=0$ describes a surface in instantaneous equilibrium without any thermal conduction into the sub-surface, while $\Gamma=2500 \mathrm{~J} \mathrm{~m}^{-2} \mathrm{~s}^{-0.5} \mathrm{~K}^{-1}$ corresponds to a highly conductive solid granite surface without any dust regolith. The Moon, with it thick highly insulating dust layer, has a thermal inertia of $39 \mathrm{~J} \mathrm{~m}^{-2} \mathrm{~s}^{-0.5} \mathrm{~K}^{-1}$ (Keihm 1984).

This wide range of thermal inertias has no big impact on the resulting weighted mean diameter and albedo values: A $\Gamma=0$ would give weighted mean values of $D_{\text {eff }}=0.30 \mathrm{~km}$ and $p_{\mathrm{V}}=0.24$, while a $\Gamma=2500$ would give $D_{\text {eff }}=0.33 \mathrm{~km}$ and $p_{\mathrm{V}}=0.19$. But the standard deviation of the 20 diameter (or albedo) values changes enormously (more than a factor of 2) for different thermal inertias. Figures 3 and 4 illustrate this effect on basis of the $\sigma_{\text {albedo }} /$ albedo and $\sigma_{\text {diameter }} /$ diameter values. A thermal inertia of $\Gamma=1000 \mathrm{~J} \mathrm{~m}^{-2} \mathrm{~s}^{-0.5} \mathrm{~K}^{-1}$ would therefore give the best match with our complete observational data set (dashed lines). We repeated the whole optimisation process with the highest quality data only (excluding the data from April, 9th, 2001, M. Delbo, priv. comm.). The resulting best thermal inertia would then be at around $750 \mathrm{~J} \mathrm{~m}^{-2} \mathrm{~s}^{-0.5} \mathrm{~K}^{-1}$ (solid line). A last robustness check with only 9 observations (dotted line) confirmed this solution.

In a second iteration, we tested the beaming model, parameterised by $\rho$, the rms of the surface slopes, and $f$, the fraction of surface covered by craters. Both parameters were kept variable between 0.1 and 0.9 (see also Dotto et al. 2000). However, the effects with $\rho$ and $f$ are not as dramatic, mainly because most of our observations were taken at large phase angles where the beaming does not play an important role. We could not find a clear minimum in the $\sigma_{\text {albedo }} /$ albedo values in the $\rho-f$ plane. Some good solutions disappeared again when we checked for robustness by using various subsets of the observational data. As a conclusion from all optimisation runs we can only say that the very smallest values $(0.1-0.3)$ are very unlikely for the beaming parameters $\rho$ and $f$ for Itokawa, all other values still seem to be in agreement with our data set. We accepted therefore the default beaming values, $\rho=0.7$ and $f=0.6$, which were derived for main-belt asteroids (Müller et al. 1999).

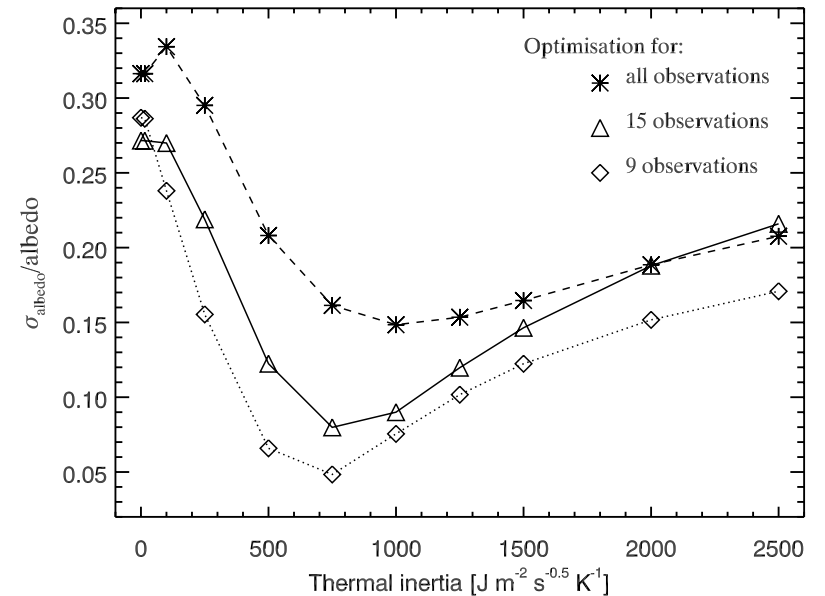

Fig. 3. Thermal inertia optimisation process for the individual TPM albedos and their standard deviation, using all 20 individual observations (dashed line), a subset with 15 observations (solid line) and a subset of 9 observations (dotted line).

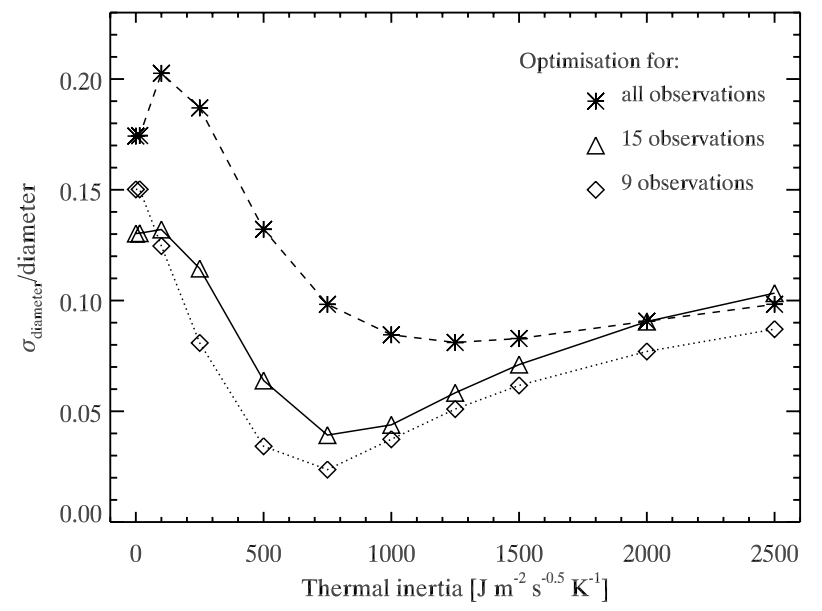

Fig. 4. Thermal inertia optimisation process for the individual TPM diameters and their standard deviation, using all 20 individual observations (dashed line), a subset with 15 observations (solid line) and a subset of 9 observations (dotted line).

As result of this optimisation process we accepted the following values:
$\Gamma=750 \mathrm{~J} \mathrm{~m}^{-2} \mathrm{~s}^{-0.5} \mathrm{~K}^{-1}$, thermal inertia
$\rho=0.7 \quad$ rms of the surface slopes
$f=0.6$ fraction of surface covered by craters.

Using the above parameters we derived the weighted mean values for $D_{\mathrm{eff}}$ and $p_{\mathrm{V}}$ together with the standard deviations ${ }^{1}$ :

$$
\begin{aligned}
& D_{\text {eff }}=0.32 \pm 0.03 \mathrm{~km}( \pm 0.01 \mathrm{~km}) \\
& p_{\mathrm{V}}=0.19 \pm 0.03( \pm 0.01) .
\end{aligned}
$$

\section{Thermophysical modelling of the new dataset}

We also used the TPM on basis of the established parameters to "transport" or normalise the observed fluxes to a reference wavelength of $10.0 \mu \mathrm{m}$. Here we concentrated on the

\footnotetext{
1 Accepting the results from the 15 highest quality data only decreases the standard deviations significantly (given in brackets).
} 


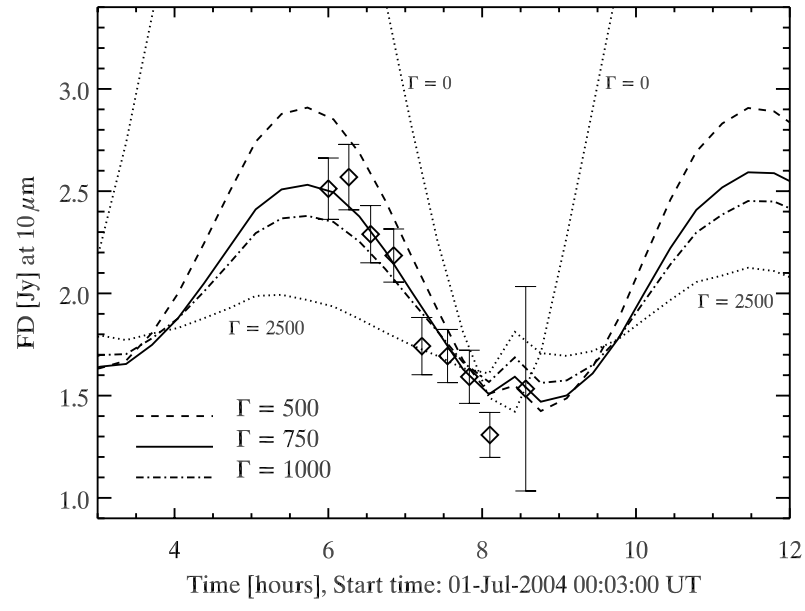

Fig. 5. Predicted thermal lightcurve at $10.0 \mu \mathrm{m}$ for the time period around the July 1st, 2004 observations. The original measurements were "transported" to the $10.0 \mu \mathrm{m}$ wavelength via our TPM solution. Predictions and measurements are shown with their absolute values, no shifting or scaling in time or flux have been done.

third dataset which has a $2.5 \mathrm{~h}$ coverage of the $12.1 \mathrm{~h}$ rotation period. For each of the measured values in Table 3 we calculated the corresponding $D_{\mathrm{eff}}$ and $p_{\mathrm{V}}$ values (for $\Gamma=$ $750 \mathrm{~J} \mathrm{~m}^{-2} \mathrm{~s}^{-0.5} \mathrm{~K}^{-1}, \rho=0.7, f=0.6$ ). This was then used again to predict the $10.0 \mu \mathrm{m}$ brightness for the given epoch (see diamond-symbols in Fig. 5). A weighted average $D_{\text {eff }}$ and $p_{\mathrm{V}}$ (of dataset \#3) was then taken to predict the thermal lightcurve (solid, dashed, dashed-dotted, and dotted lines in Fig. 5), based on the Kaasalainen shape and spin-vector model and different thermal inertia values.

Figure 5 confirms that the thermal inertia has to be somewhere in the range between 500 and $1000 \mathrm{~J} \mathrm{~m}^{-2} \mathrm{~s}^{-0.5} \mathrm{~K}^{-1}$. It also demonstrates that the implementation of the shape model in combination with the spin vector and zero points in time and phase yields consistent results. Our observational data cover the rotational phases between $354.9^{\circ}$ (14-Mar.-2001 05:50:00 UT) and 71.1 (01-Jul.-2004 08:37:00 UT).

\section{Discussion}

All optimisation steps work well under the assumption that the shape and spin vector solutions are of good quality and that the albedo is the same all over the asteroid surface, e.g. for large main-belt asteroids which have almost spherical shapes with a very homogeneous albedo distribution due to a thick dust regolith. The shape model of Itokawa matches the visual lightcurves taken at very large range of phase angles and different observing and illumination geometries (Kaasalainen et al. 2005). Kaasalainen et al. (2003) detected no significant albedo variegations. Thus, both prerequisites are fulfilled and it was for the first time possible to extract thermal properties for such an elongated object. So far, this was only possible for large main-belt asteroids (e.g. Spencer et al. 1989; Müller \& Lagerros 1998) and a few NEAs (e.g. Harris \& Davies 1999) with almost spherical shapes where the radiometric diameter/albedo solutions were not affected too much by shape effects or albedo deviations at certain epochs.
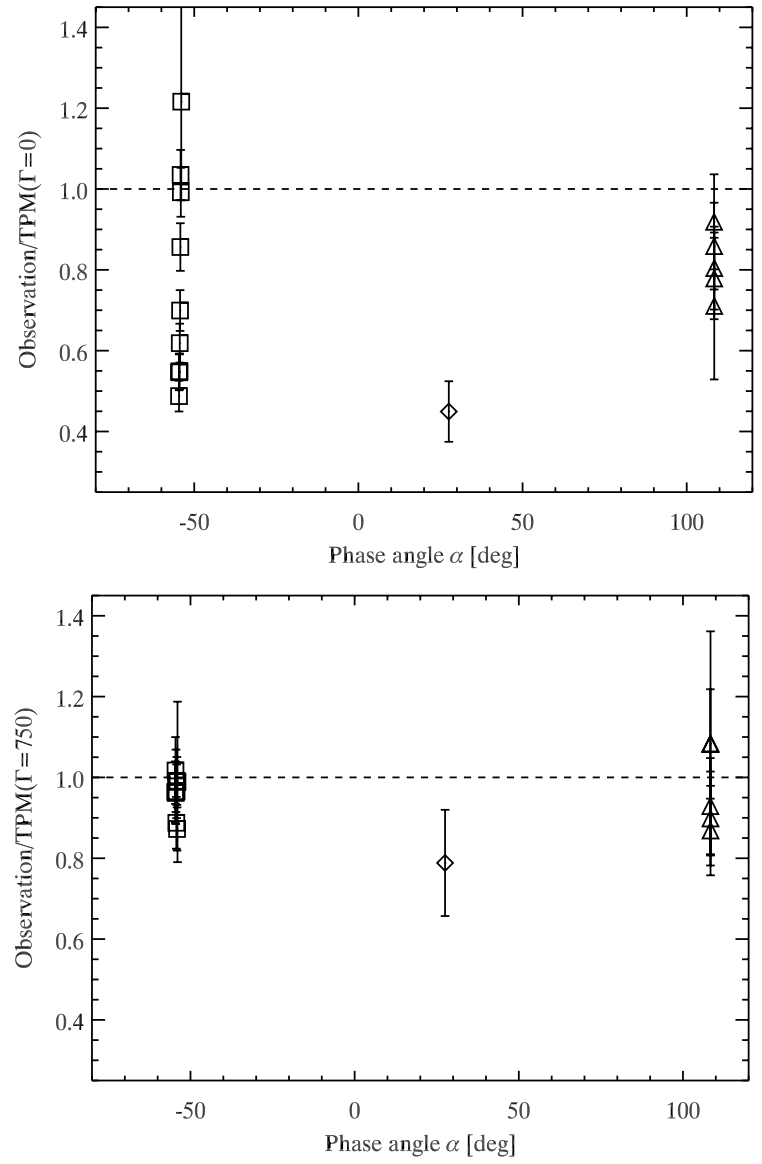

Fig. 6. The observation/TPM ratios for a thermal inertia of 0 (top) and 750 (bottom). The high thermal inertia values eliminate the trend with phase angle and reduce the scatter significantly.

Another key element for a successful derivation of thermal parameters is the wavelength and phase angle coverage of the observational data set. In order to separate beaming and thermal inertia effects, it is necessary to have equal quality data at different phase angles. An often used indicator for reasonable model assumptions is the ratio plot "observation/model prediction" (e.g. Müller \& Blommaert 2004).

Figure 6 shows this "obs/mod"-ratio plot for the phase angles. Asymmetries in the phase angle plot (see Fig. 6, top) confirm the retrograde sense of rotation (see e.g. Müller 2002 for further discussions) and show that the terminator has very different temperatures before $(\alpha>0)$ and after opposition $(\alpha<0)$, resulting in a poor modelling (large scatter) in the after opposition ratios. The TPM requires therefore a higher thermal inertia to match the observed fluxes (Fig. 6, bottom) and to eliminate the phase angle asymmetry.

Figure 7 shows how the "obs/mod"-ratio varies with wavelengths. In case of a low thermal inertia (top), one can clearly see a trend of the ratio with wavelength. For the $\Gamma=750$-case, the scatter of the data points is much smaller and the trend with wavelength disappeared. Note, that the data points, which are considered to be less reliable (Delbo, priv. comm.), are not shown for clarity reasons.

Our derived albedo of $p_{\mathrm{V}}=0.19 \pm 0.03$ fits nicely within the established S-type albedo range of $0.21 \pm 0.07$ 

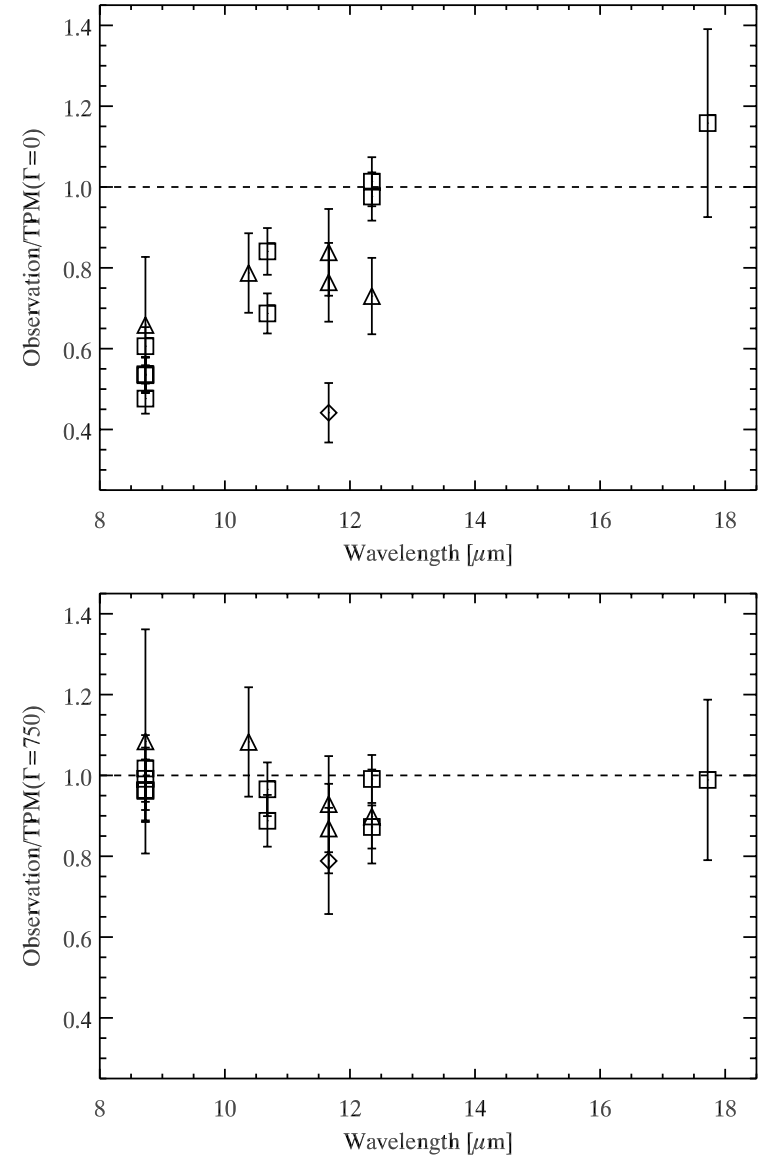

Fig. 7. The observation/TPM ratios for a thermal inertia of 0 (top) and 750 (bottom). The high thermal inertia values eliminate the trend with wavelength and reduce the scatter significantly.

(Ishiguro et al. 2003). But this albedo value is strongly connected to the $H_{\mathrm{V}}$-value of $19.9 \mathrm{mag}$. A change of $0.1 \mathrm{mag}$ in $H_{\mathrm{V}}$ to $19.8 \mathrm{mag}$ would increase the radiometric albedo by about 0.02 , while the effective diameter would remain practically unchanged. Even very large modifications of the $H_{\mathrm{V}^{-}}$ value by e.g., $0.5 \mathrm{mag}$ to $H_{\mathrm{V}}=19.4 \mathrm{mag}\left(H_{\mathrm{R}}=19.0 \mathrm{mag}\right.$ from Nishihara et al. 2005 and $(V-R)=0.4$ mag from Lowry et al. 2005 ) would lead to a marginally increased $D_{\text {eff }}=0.33 \mathrm{~km}$. But in this case, the directly connected albedo would increase to $p_{\mathrm{V}}=0.29$. Based on these considerations, we give a final solution of $p_{\mathrm{V}}=0.19_{-0.03}^{+0.11}$ to account for the different published $H_{\mathrm{V}}$ values. This shows that the size determination is closely coupled to the quality of the thermal photometry, while the albedo depends much more on the properties from the reflected light analysis, i.e., the values in the $H-G$ magnitude system. It should also be noted here that the $H-G$ concept is a badly defined convention for irregular bodies. The $H_{\mathrm{V}}=19.9 \mathrm{mag}$ is the best possible solution in the context of the Kaasalainen et al. (2005) shape model. Nishihara et al. (2005) also determined a larger $G$-value of 0.25 (instead of the 0.21 used here). But this difference would no be noticeable in the radiometric results. We also checked the robustness of our $\Gamma$-solution against uncertainties in the $H-G$ values. But taking the Nishihara et al. (2005) $H-G$ values ( $H=19.4 \mathrm{mag}, G=0.25$ ) leads to the same, very pronounced minimum at around $750 \mathrm{~J} \mathrm{~m}^{-2} \mathrm{~s}^{-0.5} \mathrm{~K}^{-1}$ in the $\sigma_{\text {albedo }} /$ albedo picture (see Fig. 3).

The derived radiometric value $D_{\text {eff }}$ is the diameter of an equal volume sphere, based on the Kaasalainen-shape and spinvector model. A rotating ellipsoid approximation can be described by the absolute sizes $2 a, 2 b$ and $2 c$, corresponding roughly to the $x-, y-$ and $z$-dimensions of Fig. 2 (longest extension, hight in bottom and hight in top image):

$2 a=0.52 \pm 0.05 \mathrm{~km} ; 2 b=0.27 \pm 0.03 \mathrm{~km} ; 2 c=0.23 \pm 0.02 \mathrm{~km}$

note however, that for very general shapes, like the Itokawashape by Kaasalainen et al. (2005), the $a / b$ and $b / c$ ratios are not well defined.

The radar observations (Ostro et al. 2005) resulted in a size estimate of594 $\times 320 \times 288 \mathrm{~m}( \pm 10 \%)$, based on the same lightcurve-based spin vector by Kaasalainen et al. (2005). The axis ratios of the lightcurve and radar shape models agree and have approximate values of $a / b=1.9$ and $b / c=1.1^{2}$. But the effective diameter $D_{\text {eff }}=2(a b c)^{1 / 3}$ of the radar solution is about $15 \%$ higher. We tried to use the radar effective diameter of about $0.38 \mathrm{~km}$ to fit the observed highest quality data from July 1, 2004. Only with very unrealistic assumptions of $p_{\mathrm{V}}=0.5$ and a thermal inertia $\Gamma=5000 \mathrm{~J} \mathrm{~m}^{-2} \mathrm{~s}^{-0.5} \mathrm{~K}^{-1}$ the TPM predictions would provide an acceptable match with the observed fluxes. Taking the stated radar uncertainty of $\pm 10 \%$ into account, we conclude that the radar sizes are overestimated by a few percent.

Sekiguchi et al. (2003) derived through the NEATM (Harris 1998) radiometric diameter and albedo values which agree within the specified error bars with our solution. The NEATM results for the NEA 2002 NY40 (data at one phase angle only) also compare well with the TPM predictions (Müller et al. 2004). But for data sets covering very different phase angles, as it is the case here, the NEATM requires different beaming parameters (Delbo et al. 2003), while the TPM can explain all observed data points with one set of physical and thermal parameters. The TPM beaming model, parameterised by $\rho$ and $f$, can handle the very different illumination geometries without artificial correction factors. Additionally, the NEATM cannot explain the before/after opposition (or morning/evening) effect, unless the beaming parameters are adjusted differently before and after opposition. A detailed comparison with the NEATM was therefore not performed.

We also tried to determine surface roughness properties, described in the TPM by $\rho$ and $f$. It turned out, that the TPM predictions for our given large phase angles are almost independent of these beaming parameters. This is in agreement with the fact that the effect only plays a role at small phase angles where mutual heating within the crater structures produce an enhanced amount of infrared flux (as compared to a smooth surface). Our phase angle coverage therefore does not allow to draw any conclusions on the surface roughness, crater structures or rms values of the surface slopes. Additional data at

\footnotetext{
2 Note here again that the dimensions are for a rough and not well-defined ellipsoid representation rather than, e.g., for the largest extents.
} 
small phase angles close to opposition are required for such investigations.

We also compared the difference between using a spherical shape model (together with the true spin vector solution under the given observing geometries in combination with the TPM) and using the Kaasalainen shape model. Assuming a spherical shape gives in fact very similar mean (or weighted mean) diameter and albedo values, but the scatter between the 20 derived albedo values is about $50 \%$ larger. The derived diameter and albedo values are then dependent on the rotational phase and to a certain extent also on the aspect angle. Additionally, the effective diameter and albedo one obtains by simply averaging our observations is not significantly different than that derived from the rotationally resolved observations, since they span a range more or less uniformly from maximum to minimum of the lightcurve, as shown in Fig. 5. The observations in July 2004 allowed to determine a $10.0 \mu \mathrm{m}$ lightcurve which is perfectly matched by the Kaasalainen et al. (2005) shape and spin vector model together with the TPM and a thermal inertia of $750 \mathrm{~J} \mathrm{~m}^{-2} \mathrm{~s}^{-0.5} \mathrm{~K}^{-1}$. In general, measurements taken at relatively large phase angles after opposition (here $\alpha=-54^{\circ}$ ), where the terminator is still warm (for an object with retrograde rotation), can be considered as key observations to determine thermal properties of NEAs. The only important point is a sufficient coverage of the rotational phases. The measurements before opposition are much more influenced by the actual illumination and observing geometry and only in second order by the contribution from the cold terminator.

The thermal inertia is defined as $\Gamma=\sqrt{\kappa_{\mathrm{s}} \rho_{\mathrm{s}} c_{\mathrm{s}}}$, with $\kappa_{\mathrm{S}}$ being the thermal conductivity, $\rho_{\mathrm{s}}$ the density and $c_{\mathrm{s}}$ the heat capacity of the surface material. A dust layer on the Itokawa surface, like the one on the Moon, with typical Moon-like $\kappa_{\mathrm{S}}$ and $c_{\mathrm{S}}$ values (Keihm 1984) would require a density several hundred times higher than the $1250 \mathrm{~kg} \mathrm{~m}^{-3}$ for the Moon to account for the derived thermal inertia. On the other hand, combining the derived high thermal inertia with the S-type bulk density of Ida (Belton et al. 1995) of $2600 \mathrm{~kg} \mathrm{~m}^{-3}$ and the specific heat of Granite $c_{\mathrm{s}}=800 \mathrm{~J} \mathrm{~kg}^{-1} \mathrm{~K}^{-1}$ the $\kappa_{\mathrm{s}}$ value would be in the order of $0.3 \mathrm{~W} \mathrm{~m} \mathrm{~m}^{-1} \mathrm{~K}^{-1}$. This seems to be a reasonable conductivity for a porous stony material. The porosity itself can be determined from the assumed bulk density of $2600 \mathrm{~kg} \mathrm{~m}^{-3}$ in combination with an anhydrous ordinary chondrite surface composition (Ishiguro et al. 2003): $p=\left(1-\rho_{\text {bulk }} / \rho\right) \sim 0.3$ (Belton et al. 1995). In conclusion, the mid-IR data are in very good agreement with the assumption of a bare rock surface. A thick dust regolith can be excluded as well as a metallic surface which would have a $\Gamma$-value above $10000 \mathrm{~J} \mathrm{~m}^{-2} \mathrm{~s}^{-0.5} \mathrm{~K}^{-1}$ and consequently produce a very small thermal lightcurve amplitude (see Fig. 5).

The total mass of Itokawa is directly connected to the assumed bulk density and the determined volume of the Kaasalainen-shape model via the $D_{\text {eff }}$ value (full uncertainty range of both quantities has been applied):

$M=\rho_{\text {bulk }} \cdot$ Volume $=2600 \frac{\mathrm{kg}}{\mathrm{m}^{3}} \cdot \frac{4}{3} \pi\left(\frac{D_{\mathrm{eff}}}{2}\right)^{3}=4.5_{-1.8}^{+2.0} \times 10^{10} \mathrm{~kg}$

\section{Conclusion}

The example of Itokawa shows the potential of the TPM applications for NEAs. State-of-the-art shape models from radar and lightcurve inversion techniques can be used for sophisticated thermo-physical investigations. In fact, the Itokawa case was the first implementation of Kaasalainen-shape models in the context of the TPM by Lagerros (1996; 1997; 1998). The TPM allows the combination of observational data taken at different observing and illumination geometries and wavelengths. No artificial fitting parameters are necessary to explain the spectral energy distributions nor the thermal behaviour with phase angle. And the thermal lightcurve is a "normal" output product for objects with known sizes and shapes.

The Hayabusa mission will characterise Itokawa's properties with high reliability. Our derived properties can therefore be compared and the TPM be verified. This project will establish the "ground truth" for future NEA TPM applications. The experience with the thermal data are very important for the planning of future observing campaigns of NEAs. Depending on the availability of a target, the wavelengths, the phase angles and the rotational phases can be selected in such a way that the thermophysical characterisation benefits most. E.g. if the surface roughness properties are of interest, one would have to include observations close to opposition. If thermal inertia is important, certain phase angles and/or a significant coverage of the thermal lightcurve are key ingredients for a successful study.

Acknowledgements. We would like to thank F. Hormuth for his support in the data analysis of the TIMMI2 observations and J. Lagerros for his modifications in the TPM code to allow a proper use of the Kaasalainen shape models. M. Delbo supported our re-evaluation of the TIMMI2 data of his thesis work.

\section{References}

Abe, M., Ohba, Y., Ishiguro, M., et al. 2002a, Lunar and Planetary Sci. Conf., 33, 1666

Abe, M., Ohba, Y., Ishiguro, M., et al. 2002b, Asteroids, Comets and Meteors, abstract, 18-24

Belton, M. J. S., Chapman, C. R., Thomas, P. C., et al. 1995, Nature, 374,785

Binzel, R. P., Rivkin, A. S., Bus, S. J., et al. 2001, Meteoritics \& Planetary Sci., 36, Suppl., p. A20

Delbó, M., Harris, A. W., Binzel, R. P., et al. 2003, Icarus, 166, 116

Delbó, M. 2004, Ph.D. Thesis, FU Berlin, http://www.diss. fu-berlin.de/2004/289/index.html

Dotto, E., Müller, T. G., Barucci, M. A., et al. 2000, A\&A, 358, 1133

Harris, A. W. 1998, Icarus, 131, 291

Harris, A. W., \& Davies, J. K. 1999, Icarus, 142, 464

Harris, A. W., \& Lagerros, J. S. V. 2002, Asteroids III, ed. W. F. Bottke Jr., A. Cellino, P. Paolicchi, \& R. P. Binzel (Tucson: University of Arizona Press), 205

Ishiguro, M., Abe, M., Ohba, Y., et al. 2003, PASJ, 55, 691

Kaasalainen, M., Kwiatkowski, T., Abe, M., et al. 2003, A\&A, 405, L29

Kaasalainen, M., Abe, M., Byron, J., et al. 2005, Proceedings of the 1st Hayabusa Symposium, ASP Conf. Ser., submitted 
Käufl, H. U., Sterzik, M., \& Siebenmorgen, R. 2003, SPIE 4841, 117 Keihm, S. J. 1984, Icarus, 60, 568

Lagerros, J. S. V. 1996, A\&A, 310, 1011

Lagerros, J. S. V. 1997, A\&A, 325, 1226

Lagerros, J. S. V. 1998, A\&A, 332, 1123

Lowry, S. C., Weissman, P. R., Hicks, M. D., et al. 2005, Icarus, 176, 408

Müller, M., Delbo, M., Kaasalainen, M., et al. 2004, The 1st HAYABUSA Symp., October 20-22, 2004, Abstract Müller, T. G. 2002, M\&PS, 37, 1919

Müller, T. G., \& Lagerros, J. S. V. 1998, A\&A, 338, 340

Müller, T. G., \& Lagerros, J. S. V. 2002, A\&A, 381, 324

Müller, T. G., \& Blommaert, J. A. D. L. 2004, A\&A, 418, 347
Müller, T. G., Lagerros, J. S. V., Burgdorf, M., et al. 1999, ESA SP-427, in The Universe as Seen by ISO, ed. P. Cox, \& M. F. Kessler, 141

Müller, T. G., Sterzik, M. F., Schütz, O., Pravec, P., \& Siebenmorgen, R. 2004, A\&A, 424, 1075

Nishihara, S., Abe, M., Hasegawa, S., et al. 2005, Lunar and Planetary Sci., XXXVI, 1833

Ostro, S. J., Benner, L. M., Nolan, M. C., et al. 2004, Met. Plan. Sci., 39, 407

Ostro, S. J., Benner, L. M., Magri, C., et al. 2005, Met. Plan. Sci., submitted

Sekiguchi, T., Abe, M., Böhnhardt, H., et al. 2003, A\&A, 397, 325

Spencer, J. R., Lebofsky, L. A., \& Sykes, M. V. 1989, Icarus, 78, 337 